\title{
ESTUDO COMPARATIVO ENTRE A AUTONOMIA FUNCIONAL DE MULHERES IDOSAS PRATICANTES E NÃO PRATICANTES DE HIDROGINÁSTICA
}

\section{Comparattive study between the functional autonomy of elderly women practicing and not practicing hydro-gymmastics}

\section{Daniel Belloni ${ }^{1}$, Alessandro Carielo de Albuquerque ${ }^{1}$, Thiago de Oliveira Rodrigues ${ }^{2}$, Mauro Lúcio Mazini Filho ${ }^{3}$, Vernon Furtado da Silva ${ }^{1}$}

\section{Resumo}

O presente estudo teve o objetivo de comparar a autonomia funcional de mulheres idosas, praticantes e não praticantes de hidroginástica. A amostra foi composta por 19 mulheres, com idades entre 61 e 80 anos, divididas em dois grupos (GPH - grupo praticante de hidroginástica, GNPH - grupo não praticante de hidroginástica). A avaliação da autonomia funcional foi realizada utilizando o protocolo GDLAM (Vale et al., 2006), constituído pelos testes de caminhar 10 metros $(\mathrm{C} 10 \mathrm{~m})$, levantar-se da posição sentado (LPS), levantar-se da posição decúbito ventral (LPDV), levantar-se da cadeira e locomover-se pela casa (LCLC). A análise estatística foi realizada por meio do teste t-Student para amostras independentes, com o nível de significância de $\alpha<0,05$. O GNPH levou o dobro do tempo para realizar as tarefas de LPS, LPDV e LCLC ao ser comparado com o GPH $(p<0,05)$. Contudo, ao desempenhar o teste de $\mathrm{C} 10 \mathrm{~m}$, o mesmo não se revelou diferente estatisticamente $(p>0,05)$. Conclui-se, baseado nos dados revelados, que a hidroginástica pode ser uma atividade física utilizada para prevenir o declínio funcional, e, ainda, conservar a autonomia funcional de mulheres idosas.

Palavras-chave: Autonomia Funcional, Atividade Física, Envelhecimento.

\begin{abstract}
This study aims to compare the functional autonomy of elderly women, practicing and not practicing hydro-gymnastics. The sample was composed of 19 women, between the ages of 61 and 80 , divided into two groups (GPH - group practicing hydro-gymnastics and, GNPH - group not practicing hydro-gymnastics). The evaluation of functional autonomy was realized using the protocol GDLAM (Vale et al., 2006), constituted by tests of walking 10 meters $(\mathrm{W} 10 \mathrm{~m})$, rise from a sitting position (RSP), rising from a ventral decumbent position (RVDP), rising from a chair and moving about the house (RCLH). The statistical analysis was realized through the test $\mathrm{t}$-Student for independent samples, with the level of significance of $\alpha<0.05$. The GNPH group took double the time to realize the tasks of RSP, RVDP and RCLH when compared with the GPH group $(p<0.05)$. However, when performing the test of $\mathrm{W} 10 \mathrm{~m}$, the same difference was not shown statistically ( $p>0.05)$. It was concluded, based on the data revealed, that hydro-gymnastics could be a physical activity utilized to prevent functional decline and, also, to conserve the functional autonomy of elderly women.
\end{abstract}

Key words: Functional Autonomy, Physical Activity, Aging.

1. Universidade Castelo Branco - Rio de Janeiro - RJ - Brasil.

2. Centro Universitário de Volta Redonda - Volta Redonda - RJ - Brasil.

3. Universidade Trás-os-Montes e Alto Douro - Vila Real - Portugal.

Recebido em 07.09.2007. Aceito em 18.01.2008.

Revista de Educação Física 2008;140:20-26 


\section{INTRODUÇÃO}

O desenvolvimento tecnológico da medicina ocasionou benefícios à população em geral, particularmente para os indivíduos com idades superiores a 65 anos, em virtude de inúmeros medicamentos que permitiram um maior controle e tratamento de doenças contagiosas e crônico-degenerativas (Yaffe et al., 2001). Em decorrência deste acontecimento, torna-se cada vez maior o número de pessoas idosas no mundo. Acredita-se que, por volta do ano 2020 , cerca de $20 \%$ da população americana terá mais de 65 anos de idade e, aproximadamente, 1,3 milhões de pessoas terão 100 anos, ou mais, próximo ao ano 2040 (César et al., 2004; Filho et al., 2006). Segundo Pereira et al. (2003), no Brasil, as estimativas dispõem uma proporção de $25 \%$ de indivíduos idosos para cada jovem, em 2050. Esse fato, sobre o envelhecimento populacional, faz emergir uma grande preocupação com a qualidade de vida dessas pessoas, uma vez que o sedentarismo é uma freqüente característica em seus estilos de vida (César et al., 2004; Filho et al., 2006).

De acordo com a Sociedade Brasileira de Medicina do Esporte (Nóbrega et al., 1999), o modo mais eficiente de promover saúde no indivíduo idoso é prevenir seus problemas clínicos mais corriqueiros. Por exemplo, evidências indicam que, ao adotar um estilo de vida mais ativo, expresso pela prática regular de exercícios físicos, poderia ser substancialmente reduzido o número de mortes ocasionadas pelo sedentarismo, tais como infarto do miocárdio (Wannamethee et al., 2000), acidente vascular encefálico (Luc et al., 2000), diabetes mellitus (Van Dam et al., 2002), bem como a hipertensão arterial (Lombardi e Parati, 2000). Além disso, sabe-se que o exercício físico regular é uma estratégia bastante eficaz para conservar a autonomia funcional de indivíduos idosos (Gardner et al., 2000; Hauer et al., 2003; Tinetti, 2003), repercutindo em um bem-estar social, psicológico e físico (Shephard e Futcher, 1997; McTierman et al., 1999; Fabrício et al., 2004; Miyoshi et al., 2004; Alberton et al., 2005), fornecendo, conseqüentemente, uma melhor qualidade de vida a essa população (Yaffe et al., 2001; Brochu et al., 2002).

Uma forma de exercício físico muito recomendada pela Sociedade Brasileira de Medicina do Esporte, para a população idosa, é a hidroginástica (Nóbrega et al., 1999). A hidroginástica proporciona inúmeros benefícios à saúde de quem a pratica. Melhoras nos níves de força, de potência aeróbia e da composição corporal são obervadas, freqüentemente, em indivíduos praticantes de hidroginástica (Miyoshi et al., 2004; Alberton et al., 2005). Além disso, à medida que ocorre o processo fisiológico do envelhecimento, indivíduos idosos, que tiveram uma vida sedentária, tendem a perder sua mobilidade, não sendo tão capazes de praticar exercícios físicos de qualquer natureza (Miyoshi et al., 2004; Alberton et al., 2005). Portanto, a prática física de exercícios realizados em meio líquido, como na hidroginástica, pode ser uma forma eficaz de proporcionar saúde, bem-estar físico, psicológico e social a indivíduos com idade avançada.

Segundo o Grupo de Desenvolvimento Latino-Americano para a Maturidade (Vale et al., 2006), é indispensável conservar a autonomia funcional em indivíduos idosos, pois, desta forma, atenuaria significativamente as oportunidades de institucionalização clínica ocorridas pela incapacidade física, além de gerar uma maior independência em suas atividades cotidianas diárias, como, por exemplo, se banhar e/ou trocar de roupa, bem como caminhar e/ou subir degraus. Portanto, as intervenções designadas para prevenir o declínio da capacidade funcional em indivíduos idosos têm uma preocupação real de, não somente gerar economia no cuidado da saúde dos idosos, mas, ainda, promover uma melhor qualidade de vida.

Um dado importantíssimo e bastante estudado sobre a fisiologia do envelhecimento demonstra que, a partir dos 60 anos, o agravamento das alterações fisiológicas e a perda das capacidades funcionais básicas (sarcopenia, ataxia, entre outros) encontram-se completamente instaladas e em velocidade extremamente acentuada. Desta forma, alguns investigadores consideram os indivíduos com as idades de 60 anos ou mais como indivíduos idosos (César et al., 2004; Vale et al., 2006).

Recentes investigações constataram que indivíduos idosos podem se beneficiar, substancialmente, de exercícios físicos, tais como a caminhada, o treinamento de força e alguns tipos de esporte, como a hidroginástica, aumentando os níveis de força, de resistência, de equilíbrio e, principalmente, de mobilidade, prolongando sua independência funcional, permitindo-lhes viver de maneira auto-suficiente e digna (ACSM, 1998; Nobrega et al., 1999; Yaffe et al., 2001; Brochu et al., 2002; Gill et al., 2002; Tinetti, 2003; César et al., 2004; Filho et al., 2006).

Desta forma, o presente estudo teve como objetivo comparar a autonomia funcional de idosos do gênero feminino, praticantes e não praticantes de hidroginástica. 


\section{METODOLOGIA}

\section{Amostra}

Dezenove mulheres, pertencentes a dois grupos (praticantes e não praticantes de hidroginástica), participaram de uma bateria de testes para um estudo comparativo sobre a autonomia funcional. Para participar do estudo, todas as voluntárias responderam negativamente aos itens do questionário PAR-Q e foram informadas dos procedimentos metodológicos da pesquisa, incluindo os possíveis desconfortos, riscos e benefícios do estudo, antes de assinarem o termo de consentimento livre e esclarecido, de acordo com as normas da resolução 196/96, do Conselho Nacional de Saúde, sobre pesquisa envolvendo seres humanos.

Foi adotado, como critério de inclusão, que as participantes do estudo deveriam ter idade igual ou superior a 60 anos e estarem completamente hábeis fisicamente para desempenharem a bateria de testes inerentes à avaliação da autonomia funcional, bem como serem independentes na execução de atividades físicas cotidianas. Como critério de exclusão, as participantes não poderiam apresentar qualquer condição aguda ou crônica que comprometesse a realização dos testes ou sua saúde física.

\section{Procedimento de coleta de dados}

Para a avaliação da autonomia funcional das participantes, foi utilizado os testes do protocolo GDLAM (Vale et al., 2006), constituídos do teste de caminhar 10 metros $(\mathrm{C} 10 \mathrm{~m})$, teste de levantar-se da posição sentada (LPS), teste de levantar-se da posição decúbito ventral (LPDV), e, ainda, teste de levantar-se da cadeira e locomover-se pela casa (LCLC). No dia em que os testes foram realizados, foi pedido para as mulheres dos dois grupos que comparecessem ao local com trinta minutos de antecedência do horário marcado para a aplicação do protocolo.

Para a realização dos testes, foi utilizada uma cadeira sem apoio para os braços, com $50 \mathrm{~cm}$ de distância do solo para o assento, um colchonete, dois cones, um cronômetro Moviment, com precisão de dois dígitos, e uma fita métrica Sanny.

\section{Tratamento experimental}

O grupo de idosas não praticantes de hidroginástica foi composto por nove idosas $(71,7 \pm 7$ anos), isentas de qualquer exercício físico direcionado há, pelo menos, um ano anterior à data da admissão na pesquisa.

O grupo de idosas praticantes de hidroginástica era composto por 10 mulheres (67,8 \pm 6 anos), participantes de um programa de hidroginástica, por um período de, pelo menos, um ano, com freqüência de três vezes por semana e duração de 50 minutos, utilizando aparelhos (acquafins, luvas e halteres) para aumentar a resistência da água, caracterizando um trabalho de potência muscular.

\section{Análise estatística}

A análise dos dados foi realizada utilizando-se o pacote estatístico SPSS 12.0. Os resultados são apresentados com médias e desvio padrão de média. A análise estatística inferencial foi desempenhada pelo teste t-Student para amostras independentes, objetivando verificar a existência de diferenças significativas de ambos os grupos e diferenças significativas entre os testes de autonomia funcional do GDLAM. O teste Kolmogorov-Smirnov foi utilizado para verificar a normalidade amostral. Para todos os procedimentos, adotou-se um intervalo de confiança de $5 \%(\alpha<0,05)$, identificando-se, assim, o ponto das direrenças reveladas.

\section{RESULTADOS}

A TABELA 1 fornece a estatística descritiva na comparação entre a idade de ambos os grupos. Pode-se observar que não houve diferença significativa entre os dois grupos.

A TABELA 2 apresenta a comparação dos testes do protocolo GDLAM e a distribuição da normalidade pelo método Kolmogorov-Smirnov entre os grupos. Os resultados do teste (GDLAM) são representados em segundos. Notou-se que houve diferença significativa $(p<0,05)$ entre a autonomia funcional nos testes de LPS, LPDV e LCLC, respectivamente. Apenas o teste de C10m não mostrou diferença significativa em ambos os grupos.

Observa-se, ainda, que o GPH, nos testes funcionais, atingiu a classificação de "bom" ou "muito bom". No entanto, o GNPH, contraproducentemente, alcançou, em apenas um dos testes de autonomia funcional, a classificação de nível "bom", sendo que os resultados restantes obtidos alcançaram classificações de nível "regular" e "fraco".

A apresentação das classificações por conceitos, de ambos os grupos, de acordo com o padrão de avaliação GDLAM, é exibido na TABELA 3. 
TABELA 1

COMPARAÇÃO ENTRE A IDADE DOS GRUPOS.

\begin{tabular}{lccc}
\hline GRUPOS & Média e DP(anos) & Máx & Mín \\
\hline GPH & $67,8 \pm 6$ & 76 & 61 \\
GNPH & $71,7 \pm 7$ & 80 & 61 \\
\hline
\end{tabular}

$\left({ }^{*} p>0,05\right)$

GPH - Grupo praticante de hidroginática; GNPH - Grupo não praticante de hidroginástica

TABELA 2

COMPARAÇÃO ENTRE OS TESTES DE AUTONOMIA FUNCIONAL E TESTE DE NORMALIDADE AMOSTRAL.

\begin{tabular}{lccccc}
\hline GRUPOS & $\begin{array}{c}\text { C10m } \\
\text { (segundos) }\end{array}$ & $\begin{array}{c}\text { LPS } \\
\text { (segundos) }\end{array}$ & $\begin{array}{c}\text { LPDV } \\
\text { (segundos) }\end{array}$ & $\begin{array}{c}\text { LCLC } \\
\text { (segundos) }\end{array}$ & $\begin{array}{c}\text { IDADE } \\
\text { (anos) }\end{array}$ \\
\hline GPH & $5,17 \pm 0,9$ & $6,65 \pm 1,7^{*}$ & $2,24 \pm 0,6^{*}$ & $37,54 \pm 4,1^{*}$ & $67,8 \pm 6$ \\
GNPH & $5,82 \pm 0,7$ & $12,28 \pm 2,7^{*}$ & $4,01 \pm 1,0^{*}$ & $49,35 \pm 6,7^{*}$ & $71,7 \pm 7$ \\
Valor de P & 0,105 & 0,000 & 0,001 & 0,000 & 0,132 \\
Distribuição & Normal & Normal & Normal & Normal & Normal \\
\hline
\end{tabular}

$\left({ }^{*} \mathrm{p}<0,05\right)$

GPH - Grupo praticante de hidroginástica; GNPH - Grupo não praticante de hidroginástica; C10m - Caminhar 10 metros; LPS - Levantar-se da posição sentada; LPDV - Levantar-se da posição decúbito ventral; LCLC - Levantar-se da cadeira e locomover-se pela casa

TABELA 3

COMPARAÇÃO ENTRE OS TESTES DE AUTONOMIA FUNCIONAL POR CONCEITO DE ACORDO COM O PROTOCOLO GDLAM.

\begin{tabular}{lccccc}
\hline Grupos & N & C 10m & LPS & LPDV & LCLC \\
\hline GPH & 10 & Muito Bom & Muito Bom & Muito Bom & Bom \\
GNPH & 9 & Bom & Fraco* & Regular $^{*}$ & Fraco* $^{*}$ \\
\hline
\end{tabular}

$\left({ }^{*} \mathrm{p}<0,05\right)$

GPH - Grupo praticante de hidroginástica; GNPH - Grupo não praticante de hidroginástica; C10m - Caminhar 10 metros; LPS - Levantar-se da posição sentada; LPDV - Levantar-se da posição decúbito ventral; LCLC - Levantar-se da cadeira e locomover-se pela casa

\section{DISCUSSÃO}

Embora um grande número de estudos tenha demonstrado os efeitos positivos dos exercícios físicos de natureza cardiovascular e, principalmente, neuromuscular, na prevenção do declínio funcional e na manutenção dos níveis autonômicos de indivíduos idosos (Nóbrega et al., 1999; Yaffe et al., 2001; Brochu et al., 2002; Gill et al., 2002; Tinetti, 2003), poucos trabalhos foram encontrados com o propósito de examinar o comportamento fisiológico de indivíduos com idade superior a 60 anos, nos exercícios em meio líquido, como, por exemplo, na hidroginástica. Assim sendo, como no estudo de Taunton et al. (1996), este estudo fornece uma forte evidência de que um programa de ginástica, realizado em meio líquido, é bastante eficaz na perspectiva de prevenir o declínio funcional e conservar a autonomia de pessoas idosas.

Ao analisar os dados sobre a caminhada de 10 metros (C10m), no grupo de idosas praticantes de hidroginástica (GPH) e no grupo de idosas não praticantes (GNPH), pôde-se verificar que os resultados atingiram médias bastante similares. Isto pode ser explicado, basicamente, pelas características deste teste funcional, pois, apesar do GNPH se encontrar em uma condição de sedentarismo, não revelaram possuir qualquer dificuldade na realização 
do teste $\mathrm{C} 10 \mathrm{~m}$, expondo terem muita facilidade em desempenhá-lo. Isto ocorreu pelo fato do padrão hábil-motor de caminhar já fazer parte do cotidiano dessas mulheres. Contuto, nos testes de levantar da posição sentada (LPS), levantar-se da posição decúbito ventral (LPDV) e levantar-se da cadeira e locomover-se pela casa (LCLC), o GNPH levou o dobro do tempo, se comparado ao GPH, para a realização dos testes, apresentando uma diferença em alta condição de significância em todos os subseqüentes testes $(p=0,0001=p<0,05)$. A diferença no tempo entre os grupos, nos testes de LPS, LPDV e LCLC, naturalmente, demonstra que indivíduos idosos mais ativos conseguem conservar sua autonomia funcional por um período mais prolongado, adiando, desta forma, os riscos de declínio funcional. Estes resultados são corroborados com o estudo realizado por Vale et al. (2006), que encontrou respostas similares para a realização destes testes. Outro dado de interesse primordial foi revelado por Pereira et al. (2003), em seus estudos, ao mostrar que idosos institucionalizados apresentaram tempos extremamentes superiores dos constatados no referido estudo, principalmente quando comparados aos tempos alcançados pelos grupos de idosas ativas. Isto, indubitavelmente, sugere que o idoso que reside no seu lar pode ser mais ativo do que aquele que reside internado em uma instituição ou em um hospital.

O decrésimo na capacidade dos músculos em produzir força rápida pode afetar, adversamente, a capacidade de adultos mais velhos desempenharem atividades como subir escadas, caminhar, levantar de uma cadeira, ou seja, desempenhar tarefas rotineiras. Alguns fatores, como, por exemplo, a redução da massa muscular, as mudanças no mecanismo de controle do sistema nervoso, as mudanças hormonais, a nutrição pobre e, principalmente, a inatividade física, podem contribuir significativamente para o declínio funcional, causando dependência física.

Taaffe (2006), em um artigo de revisão, menciona a sarcopenia como um declínio de massa muscular, provocando perda da perfomance físico-funcional na autonomia e na qualidade de vida do idoso. Nesse artigo, o autor revela que o treinamento de resistência, ou o treinamento de força, tem sido indicado como um estimulador físico, interrompendo o declínio funcional e provendo, substancialmente, uma função física. Cita, ainda, que treinar uma ou duas vezes por semana, atingindo os maiores grupos musculares em uma intensidade moderada, é o suficiente para melhorar a qualidade de vida. Taaffe (2006) afirma, ainda, que praticar exercícios físicos de qualquer natureza pode ser uma maneira definitivamente significativa para prevenir a perda das capacidades físico-funcionais causadas pelo processo de envelhecimento. Deste modo, pode-se observar que o grupo de idosas ativas, isto é, as praticantes de hidroginástica, obtiveram escores muito superiores nos testes que tangem a autonomia funcional. Portanto, corroborando com a afirmação de Taaffe (2006), idosos ativos conseguem manter sua autonomia funcional por muito mais tempo e, conforme os dados revelados por este estudo, a ginástica realizada em meio liquído parece ser uma eficaz maneira de manter a prática contínua de exercícios físicos para indivíduos idosos.

Em síntese, este estudo demonstrou que um programa de exercícios físicos direcionados, realizados na água, como a hidroginástica, pode ser uma estratégia eficaz para manter a autonomia funcional de mulheres idosas. Contudo, devido ao design transversal e a metodologia utilizada, fica bastante difícil comprovar se realmente os resultados encontrados foram proporcionados pela hidroginástica. Por exemplo, o percentual de gordura dos indivíduos não foi mensurado a fim de se indentificar uma maior homogeneidade entre os grupos. Sabe-se que esta variável pode interferir substancialmente em grande parte das tarefas inerentes ao estudo, mas, entretanto, o valor da gordura percentual não foi mensurado. Ainda, não houve adequada avaliação do status de atividade física atual conseqüente do hábito diário de vida. Tal variável, se verificada por meio de um questionário validado, poderia revelar a existência de diferenças no padrão de vida dos idosos, podendo contribuir para conclusões mais fidedignas. Outra variável que pode ter interferido nos escores do presente estudo foi a pequena diferença entre a média das idades dos indivíduos. Por exemplo, o GNPH era composto por um individuo a menos, e, ainda, possuía um maior quantitativo de indivíduos mais velhos.

Portanto, aconselha-se o desenvolvimento de mais estudos, que devem ser realizados na perspectiva de verificar os reais benefícios da prática regular da hidroginástica para indivíduos idosos. Embora o presente estudo tenha falhado em controlar algumas variáveis que pudessem interferir significativamente nos dados revelados, os escores obtidos mostraram uma forte evidência de que a hidroginástica pode melhorar os níveis 
de autonomia funcional de idosos em idade crítica de declínio funcional.

\section{CONCLUSÃO}

De acordo com os dados revelados no presente estudo, pôde-se verificar que mulheres idosas, não praticantes de hidroginástica, alcançaram um nível muito baixo, se comparadas com as praticantes de hidroginástica, em se tratando da autonomia funcional, em grande parte dos testes do protocolo GDLAM. Desta maneira, o estudo demonstrou que o grupo de idosas ativas, isto é, praticantes de hidroginástica, obteve melhores resultados nos testes do protocolo GDLAM, principalmente quando comparadas às idosas inativas. Cabe ressaltar, ainda, que a metodologia utilizada não foi a mais adequada para se confirmar que a hidroginástica é uma estratégia eficaz para manter a autonomia funcional. Portanto, mais estudos devem ser realizados na perspectiva de se verificar e identificar quais seriam os potenciais benefícios da hidroginástica para indivíduos idosos que procuram manter seus níveis de autonomia funcional.

\section{REFERÊNCIAS BIBLIOGRÁFICAS}

ALBERTON CL, COERTJENS M, FIGUEIREDO PAP, KRUEL LFM. Behavior of oxygen uptake in water exercises performed at different cadences in and out of water. Med Sci Sports Exerc 2005;37(5):S103.

AMERICAN COLLEGE OF SPORTS MEDICINE. Position Stand: the recommended quantity a quality of exercise for developing and maintaining cardiorespiratory and muscular fitness, and flexibility in health adults. Med Sci Sports Exerc 1998;30:975-91.

BROCHU M, SAVAGE P, LEE M, DEE J, CRESS ME, POEHLMAN ET et al. Effects of resistance training on physical function in disabled women with coronary heart disease. J Appl Physiol 2002;92:672-8.

CÉSAR EP, ALMEIDA OV, PERNAMBUCO CS, VALE RGS, DANTAS EH. Aplicação de quatro testes do protocolo GDLAM. Rev Minei Ed Física 2004;12(1):18-37.

FABRÍCIO SC, RODRIGUES RA, JÚNIOR ML. Causas e conseqüências de quedas de idosos atendidos em hospital público. Rev Saúde Pública 2004;38(1):93-9.

FILHO ML, FERREIRA WR, CÉSAR EP. Os benefícios do treinamento de força no desempenho da autonomia funcional do idoso. Rev de Ed Física Exército 2006;134:57-68.

GARDNER MM, ROBERTSON MC, CAMPBELL AJ. Exercise in preventing and fall related injuries in older people: a review of randomized controlled trials. Br J Sports Med 2000 34:7-17.

GILL TM, BAKER DI, GOTTSCHALK M, PEDUZZI P, ALLORE H, BYERS A. A program to prevent functional decline in physically frail, ederly persons who live at home. N Engl J Med 2002 347;(14):1068-74.

HAUER KV, HICKS AL, McCARTNEY N. Training for muscle power in older adults: effects on functional abilities. Can J Appl Phisiol 2003;28(2):178-89.

LOMBARDI F, PARATI G. An update on: cardiovascular and respiratory changes during sleep in normal and hypertensive subjects. Cardio Res 2000;45:200-11.

LUC MD, MAITE G, JEAN FT, BENOIT M, BERNARD J, JEAN C. Outcome, cunctional autonomy and quality of life of elderly patients with a long term intensive care unit stay. Crit Care Med 2000;28:3389-95.

MCTIERMAN A, SCHWARTZ RS, POTTER J, BOWEN D. Exercise clinical trials in cancer prevention research: a call to action. Cancer Epidemiol Biomark Prevent 1999;8(1):201-6. 
MIYOSHI T, SHIROTA T, YAMAMOTO S, NAKAZAWA K, AKAI M. Effect of the walking speed to the lower limb joint angular displacements, joint moments and ground reaction forces during walking in water. Disabil Rehabil 2004;26(12):724-732.

NÓBREGA AC, FREITAS EV, OLIVEIRA MA, LEITÃO MB, LAZZOLI JK et al. Posicionamento oficial da Sociedade Brasileira de Medicina do Esporte e da Sociedade Brasileira de Geriatria e Gerontologia: Atividade física e saúde no idoso. Rev Bras Med Esporte 1999;5(6):207-11.

PEREIRA IC, ABREU FC, VITORETI AV, LÍBERO GA. Perfil da autonomia funcional em idosos institucionalizados na cidade de Barbacena. Fitness e Perform J. 2003 2(5):285-8.

SHEPHARD RJ, FUTCHER R. Physical activity and cancer: How may protection be maximized? Crit Rev Oncog 1997;8(1):219-72.

TAAFFE DR. Sarcopenia: Exercise as a treatment strategy. Aust Farm Physician 2006;35(3):130-4.

TAUNTON JE, RHODES EC, WOLSKI LA, DONELLY M, WARREN J, ELLIOT J et al. Effect of land-based and water-based fitness programs on the cardiovascular fitness, strength and flexibility of women aged 65-75 years. Gerontology 1996;42(4):204-10.

TINETTI ME. Preventing falls in elderly persons. N Engl J Med 2003;348(1):42-9.

VALE RG, BARRETO AC, NOVAES JS, DANTAS EH. Efeitos do treinamento de força e de flexibilidade sobre a autonomia e qualidade de vida de mulheres senescentes. Rev Bras Cineantropom Desempenho Hum 2006;8(4):52-8.

VAN DAM RM, SCHUIT AJ, FESKENS EJ, SEIDELL JC, KROMHOUT D. Physical activity and glucose tolerance in ederly men: zutphen elderly study. Med Sci Sports Exerc. 2002;34(7):1132-6.

WANNAMETHEE GS, SHAPER AG, WALKER MM. Physical activity and mortality in older men with diagnosed coronary heart disease. Circulation 2000;102:1358-63.

YAFFE K, BARNES D, NEVITT M, LUIS L, COVINSKI K. A prospective study of physical activity an cognitive in elderly women. Arch Intern Med 2001;161:1703-8.

\section{Endereço para correspondência:}

Daniel Teixeira Belloni
Avenida Ministro José Fabrino Baião, 304 - Thomé
Cataguases - MG - Brasil
CEP: $36774-184$
Tel.: (32) $8846-6332$
e-mail: dtbelloni @yahoo.com.br

